\title{
Importance of Hypoxia Index in FHR Monitoring
}

\author{
Kazuo Maeda* \\ Honorary professor, Department of Obstetrics and Gynecology, Tottori University Medical School, Japan \\ *Corresponding author: Kazuo Maeda, Honorary professor, Department of Obstetrics and Gynecology, Tottori University Medical School, Yonago, \\ Japan
}

Submission: 眥October 16, 2017; Published: 㘹 December 20, 2017

\begin{abstract}
Aim: Numeric objective evaluation of FHR deceleration.

Methods and results: Hypoxia index (HI) is the sum of deceleration duration (min) divided by the lowest FHR (bpm), and multiplied by 100 . As the HI was 25 in a case of FHR decelerations followed by the loss of variability and cerebral palsy (CP), and the HI was 26 in a case of repeated late decelerations for $50 \mathrm{~min}$ with the loss of variability, Apgar 3, and brain damage, the HI below 25 at delivery would be safe, showing neither brain damage nor CP, namely, the HI of 3 connected late decelerations was 6 and Apgar was 9, without CP. Also HI values of repeated abnormalities were 20-24 preserving the FHR variability, without brain damage.
\end{abstract}

Conclusion: The late, early, mild and severe decelerations and sudden acute FHR bradycardia would not be followed neither by brain damage nor $\mathrm{CP}$, when the variability is preserved , and the $\mathrm{HI}$ is lower than 25 . Thus, it is recommended to decide early caesarean delivery, when the $\mathrm{HI}$ is 20 or less, considering the time to prepare the surgery, while no normal neonate may be guaranteed by the caesarean delivery performed after the higher hypoxia index of 25 or more with the loss of FHR variability. The other sign of ominous outcome will be pathologic sinusoidal FHR, which mean severe fetal anemia

Keywords: Fetus; Hypoxia index; FHR deceleration; Late deceleration; Variable deceleration; Acceleration; Variability; Cerebral palsy; Apgar score; Long term FHR variability

\section{Introduction}

Fetal outcome of late deceleration (LD) is uncertain in fetal monitoring, namely, the outcome of its 2-3 repetition was favorable, however, LD repetition resulted severe neonatal asphyxia, associating the loss of variability and infantile brain damage, where Apgar was 3, while the threshold to be ominous outcome was unknown. Thus, the author needed new LD evaluation.

\section{Methods}

As fetal bradycardia is caused by the excited parasympathetic nerve center with hypoxia, while there was no FHR change when the animal was anesthetized [1], and apneic bradycardia disappeared and heart rate returned normal after infusion of oxygenated blood to anencephalic neonate [2], thus, fetal bradycardia is only the sign of environmental hypoxia but not the sign of fetal brain damage, which is the loss of FHR variability. As short duration of low $\mathrm{PaO}_{2}$ does not affect the brain, but the brain is damaged by long hypoxic exposure as shown in hypoxic-ischemic encephalopathy. Therefore, hypoxic effect was found in long duration of hypoxia, which was determined by the sum of durations of decelerations, where the heart rate $(\mathrm{bpm})$ is used instead of $\mathrm{PaO}_{2}$, because FHR is highly correlated $\mathrm{PaO}_{2}$ when $\mathrm{PaO}_{2}$ was lower than $50 \mathrm{mmHg}$ [1], and fetal $\mathrm{PaO}_{2}$ was less than $50 \mathrm{mmHg}$ [2]. Thus the duration of hypoxic exposure was determined by the sum of deceleration duraton, while hypoxic intensity was estimated by the inversion of nadir FHR, thus, the sum of deceleration duration (min) was divided by the lowest FHR (bpm), and multiplied by 100 to calculate the hypoxia index, namely, hypoxia index (HI) is the sum of deceleration duration divided by the nadir of FHR, and multiplied by 100 .

\section{Results}

The Apgar score of three LDs of $45 \mathrm{sec}$ lag time was 6, where the outcome was normal, while HI was 25 and 26 in two fetuses of severe asphyxia followed by the loss of variability and cerebral palsy in a case, and in another case who repeated LD for $50 \mathrm{~min}$ associated by the loss of variability followed by 3 months infant death due to brain hemorrhage, while HI was 20-24 in cases of abnormal FHR but neither followed by the loss of variability nor cerebral palsy [3]. Thus the threshold neither to develop the loss of variability nor cerebral palsy is $20-24$, and the $\mathrm{HI}$ to decide to perform early caesarean delivery will be the HI of 20 or less, after the loss of acceleration and decreased amplitude of variability to the level of $5 \mathrm{bpm}$ or less.

\section{Discussion}

As the theory of hypoxia index is adopted not only late deceleration but also to all decelerations including variable decelerations, because the HI does not evaluate the lag time but the FHR score does, and also adopted to the sudden and acute 
continuous FHR bradycardia. Therefore, fetal heart rate will be evaluated with FHR score in short period for $5 \mathrm{~min}$, while by long period is evaluated with hypoxia index in computerized FHR monitoring, where the diagnosis of pathologic sinusoidal heart rate should be added.

\section{Conclusion}

The novel hypoxia index is useful not only to evaluate late deceleration, but also early and variable decelerations without the classification of deceleration patterns, excluding the controversy FHR pattern. Continuous fetal bradycardia is also evaluated by HI, excludiing hypoxic ischemic encephalopathy. Additional necessary diagnoses will be the short term FHR score and pathologic sinusoidal FHR.

\section{References}

1. Umezawa J (1975) Studies on the relation between heart rate and $\mathrm{PaO}_{2}$ in hypoxic rabbit: a comparative study for fetal heart rate change in labor. Acta Obstet Gynecol Jpn 28: 1203-1212.

2. Maeda K, Kimura S, Nakano H (1969) Studies on fetal pathophysilogy. Acta Obstet Gynecol 8: 877-886.

3. Maeda K (2014) Modalities of fetal evaluation to detect fetal compromise prior to the development of significant neurological damage. J Obstet Gynaecol Res 40(10): 2089-2094. 